\title{
Calculation of the Electromagnetic Field Around a Microtubule
}

\author{
D. Havelka, M. Cifra
}

Microtubules are important structures in the cytoskeleton which organizes the cell. A single microtubule is composed of electrically polar structures, tubulin heterodimers, which have a strong electric dipole moment. Vibrations are expected to be generated in microtubules, thus tubulin heterodimers oscillate as electric dipoles. This gives rise to an electromagnetic field which is detected around the cells. We calculate here the electromagnetic field of microtubules if they are excited at $1 \mathrm{GHz}$. This paper includes work done for the bachelor thesis of the first author.

Keywords: Bioelectromagnetism, microtubules, endogenous, electromagnetic field of biological systems.

\section{Introduction}

Generation of an electromagnetic field is not limited to the elite class of electroexcitable cells of higher organisms. A large number of experiments indicate that living cells in general generate an electromagnetic field (EMF) in a broad frequency spectrum from $\mathrm{kHz}$ [1] through $\mathrm{MHz}[2,3,4]$ to the optical region [5]. The generating mechanism varies across the spectrum. The most likely source of cellular EMF generation in the radiofrequency region is mechanical vibration of cellular electrically polar structures. This concept was first postulated and theoretically treated by Fröhlich in the late 1960s, for a review see [6]. He proposed electrically polar longitudinal vibrations interacting with an elastic field, giving rise to coherent cellular EMF. It was estimated that oscillating units are located in the cellular membrane and oscillate in the frequency region of $10^{11}-10^{12} \mathrm{~Hz}$. After the cytoskeleton was discovered and more information on the physical properties of its subunits had been gathered, microtubules emerged as a likely candidate for generation of cellular EMF [7]. Microtubules (MTs) form part of the cytoskeleton which is present in every eukaryotic cell [8]. MTs resemble hollow rod with an outer and inner diameter of $25 \mathrm{~nm}$ and $17 \mathrm{~nm}$, respectively. MTs are composed of tubulin heterodimers, which are protein of high electric dipole moment (over 1000 Debye $\sim 10^{-26} \mathrm{C} \cdot \mathrm{m}$ ) with one component aligned partially in the axis of MT. Tubulin heterodimers stack into protofilaments which assemble further to MTs. Oscillations of microtubules have been proposed to be present from the $\mathrm{MHz}$ region to the $\mathrm{GHz}$ region $[7,9,10]$. Thus tubulin heterodimers can be approximated as oscillating electric dipoles. In this paper, we show the components of the EMF around an elementary oscillating electric dipole and calculate the consequently generated EMF around MTs.

\section{Components of the electric field and the magnetic field of the elementary oscillating electric dipole}

$$
H_{\varphi}=-\frac{I d l}{4 \pi} \mathrm{k}^{2} \sin \delta\left(\frac{1}{\mathrm{j} \mathrm{k} r}+\frac{1}{(\mathrm{j} \mathrm{k} r)^{2}}\right) e^{-\mathrm{jk} r}
$$

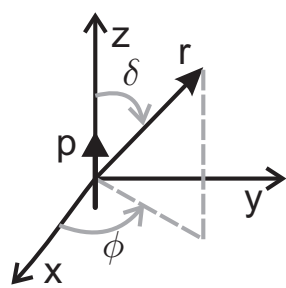

Fig. 1: A dipole in the Cartesian coordinate system

$$
\begin{array}{r}
E_{r}=-\frac{I d l}{4 \pi} Z \mathrm{k}^{2} 2 \cos \delta\left(\frac{1}{(\mathrm{j} \mathrm{k} r)^{2}}+\frac{1}{(\mathrm{j} \mathrm{k} r)^{3}}\right) e^{-\mathrm{jk} r} \\
E_{\delta}=-\frac{I d l}{4 \pi} Z \mathrm{k}^{2} \sin \delta\left(\frac{1}{\mathrm{j} \mathrm{k} r}+\frac{1}{(\mathrm{j} \mathrm{k} r)^{2}}+\frac{1}{(\mathrm{j} \mathrm{k} r)^{3}}\right) e^{-\mathrm{jk} r}
\end{array}
$$

The electromagnetic field around the elementary electric dipole (referred to as EED) is defined by equations (1-3) [11]. $H$ is magnetic field intensity, $E$ is electric field intensity, $I$ is equivalent current, $d l$ is length of the dipole, $\mathrm{k}$ is propagation constant, $Z$ is wave impedance, $\mathrm{j}$ is imaginary unit $\left(\mathrm{j}^{2}=-1\right)$ and other symbols are according to Fig. 1. None of these equations (referred to as Eqs) are dependent on $\varphi$, and all Eqs include:

$$
\mathrm{j} \omega \hat{p}=I d \vec{l}
$$

where $\omega$ is angular frequency and $p$ is dipole moment. The intensities of the electric and the magnetic fields are orthogonal to each other in every point in space. Complicated dependence of intensities with the variable $r$ denotes that the field has different properties over various distances. There are three parts of the field which differ in the value of the power of the product $\mathrm{jk} r$ in the denominator of Eqs. 1-3; i.e. near, intermediate and far zone.

\subsection{Modifications of Eqs (1-3) for calculations of the intensity modulus of the electric field in a lossy medium}

There is a dominant electrical component over a magnetic component in the near zone of EED. The near zone demonstrates dimensions far greater than the size of a cell in the frequency of $1 \mathrm{GHz}$. For this reason, we have decided to work here with the electrical component only. 
The modulus was calculated using Eqs 1-3 in a lossy medium [12]. The phase was omitted to simplify the calculation. To retain validity of the results this can be done only when the

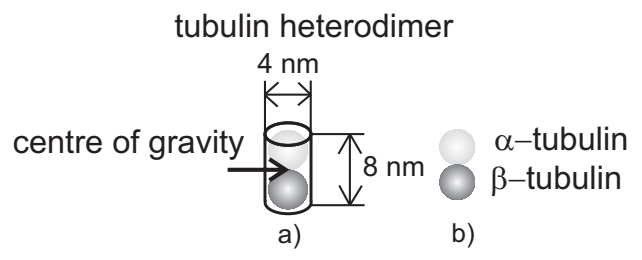

Fig. 2: An approximation of a heterodimer on EED

examined distance is much smaller than the wave length.

\section{A description of the intensity of the electric field around a microtubule}

Fig. 3 depicts a microtubule in section through the $x y$ plane. An Eq describing the calculation of angle $\varphi$ which forms an angle of axis $x$ with $D / 2$ follows:

$$
\varphi=n \frac{2 \pi}{13}
$$

where $n$ means the order of the protofilaments (referred to as PTs) and is given the values of $0,1,2$, up to 12 . The distance of a PT from the point of field evaluation (PFE) is called $R_{\mathrm{xy}}$, and will be calculated according to the following Eq:

$$
R_{\mathrm{xy}}^{2}=\frac{D^{2}}{4}+y^{2}-D y \sin \varphi \text {. }
$$

Angle $\gamma$ can be expressed as:

$$
\cos \gamma=\frac{1}{R_{\mathrm{xy}}}\left(y-\frac{D}{2} \sin \varphi\right) .
$$

The size of vector $E_{\mathrm{y}}$ is defined by the following Eq:

$$
E_{\mathrm{y}}=E_{\mathrm{xy}} \cos \gamma
$$

and using Eq. 7 we get another equation

$$
E_{\mathrm{y}}=\frac{E_{\mathrm{xy}}}{R_{\mathrm{xy}}}\left(y-\frac{D}{2} \sin \varphi\right) .
$$

An arbitrary axis $y^{\prime}$ intersecting PT and PFE will be used for calculating the contributions of each heterodimer. The cross-section of the $y^{\prime} z$ plane is shown in Fig. 4.

Components $E_{r}$ and $E_{\delta}$ are described by Eqs. 2 and 3. To simplify the calculation, $\mathrm{E}_{\delta}$ and $E_{r}$ are converted into $E_{\mathrm{xy}}$ and $E_{\mathrm{z}}$.

$$
\begin{aligned}
& E_{\mathrm{z}}=-E_{\delta} \sin \delta+E_{r} \cos \delta, \\
& E_{\mathrm{xy}}=E_{\delta} \cos \delta+E_{r} \sin \delta,
\end{aligned}
$$

where angle $\delta$ is defined by the following equation:

$$
\operatorname{tg}(\pi-\delta)=\frac{R_{\mathrm{xy}}}{h h}
$$

or

$$
\operatorname{tg} \delta=\frac{R_{\mathrm{xy}}}{h h} .
$$

Eq. 13 is used in the case that $h h<0$ (Fig. 4a) and Eq. 14 if $h h>0$ (Fig. 4b). Parameter $\boldsymbol{h} \boldsymbol{h}$ is the distance between the centre of gravity of a heterodimer from PFE, and is defined by the equation:

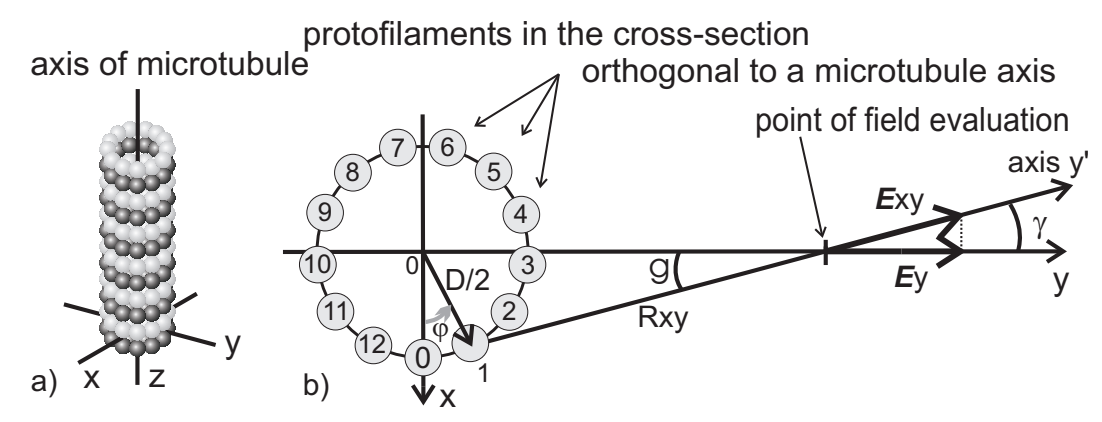

Fig. 3: a) Placement of a microtubule into the coordinate system, b) a section through the $x y$ plane [13]

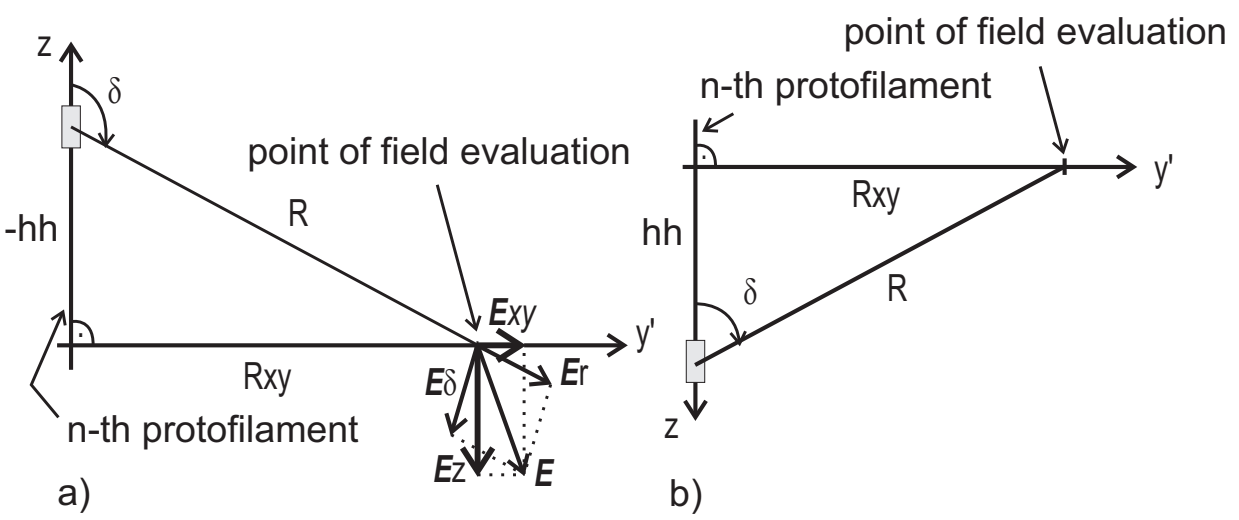

Fig. 4.: Cross-section of the $y^{\prime} z$ plane [13] 


$$
h h=z-h,
$$

where $z$ is the $z$-th coordinate PFE and $h h$ is the distance between the centre of gravity of the first heterodimer and the centre of gravity of the examined heterodimer.

$$
R=\sqrt{R_{\mathrm{xy}}^{2}+h h^{2}} .
$$

Eqs. 4-16 and 1-3 describe the intensity of the electric field of a microtubule without a shift of PT.

\subsection{Shift of protofilaments}

The lattice with intensity shift of PTs simulates a more realistic projection of the organization of PTs in the microtubule [14]. This provides more accurate information about the intensity of the electric field around MT. We have chosen the configuration in the A-lattice, which is a lattice with a longitudinal shift of PTs of $4.92 \mathrm{~nm}$ (Table 1 and Fig. 5-6).

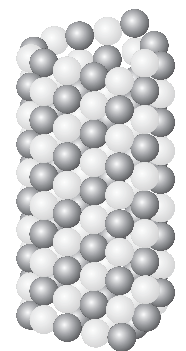

a)

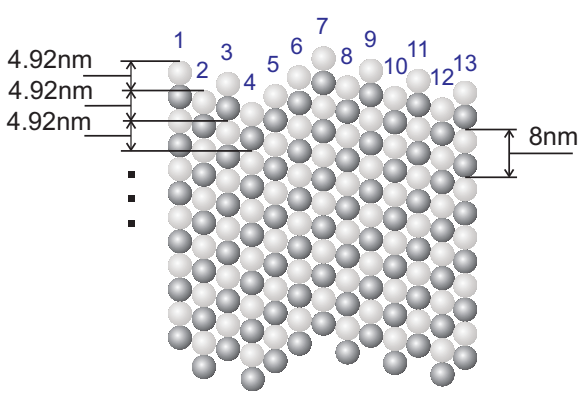

b)
Fig. 5: a) Microtubule with a longitudinal shift of 4.92nm, b) Shell of MT with a longitudinal shift of PT

Table 1: Longitudinal shift of PT in the A-lattice

\begin{tabular}{|c|c|}
\hline number of PT & shift to 1. PT $[\mathrm{nm}]$ \\
\hline 2 & -4.92 \\
\hline 3 & -1.84 \\
\hline 4 & -6.76 \\
\hline 5 & -3.68 \\
\hline 6 & -0.6 \\
\hline 7 & 2.48 \\
\hline 8 & -2.44 \\
\hline 9 & 0.64 \\
\hline 10 & -4.28 \\
\hline 11 & -1.2 \\
\hline 12 & -6.12 \\
\hline 13 & -3.04 \\
\hline
\end{tabular}

\subsection{Coherent and random excitation}

All dipoles (heterodimers) are excited in phase when excited coherently. We have also attempted to simulate incoherent (thermal) oscillations by two means, using a randomly oriented dipole moment. For the first approximation, we used a randomly generated matrix which turned the dipole moment either to positive or to negative axis $z(+-)$. For the second approximation, the matrix of random numbers from 0 to 1 was generated ( $\left.\mathrm{p}^{*} \sin \right)$. The size of the dipole moment was defined by the function:

$$
p p=p \cdot \sin (2 \pi \cdot n),
$$

where $n$ is the value of the matrix element corresponding to a certain heterodimer, and for $p$ see Table 2.

Table 2: Material constants and the dipole moment

\begin{tabular}{|l|l|}
\hline Dipole moment: & $\begin{array}{l}p=10^{-26} \mathrm{Cm} \\
(1000 \text { Debye })[14]\end{array}$ \\
\hline $\begin{array}{l}\text { Relative permittivity } \\
\text { (non-conductive medium): }\end{array}$ & $\varepsilon_{r}=81$ (water) \\
\hline $\begin{array}{l}\text { Relative permittivity (con- } \\
\text { ductive medium): }\end{array}$ & $\varepsilon_{r}=60[15]$ \\
\hline Conductivity: & $\sigma=1 \mathrm{~S} / \mathrm{m}[15]$ \\
\hline
\end{tabular}

\subsection{Depiction of the direction and magnitude of the electric field}

The size of the electric field (EF) was drawn by function surf, see e.g. Fig. 6. For depicting the direction of EF, which is a slightly more complicated function, a vectarrow [16] was used. The vector describes only the direction of EF. The magnitude of EF is expressed by color scale in the picture.

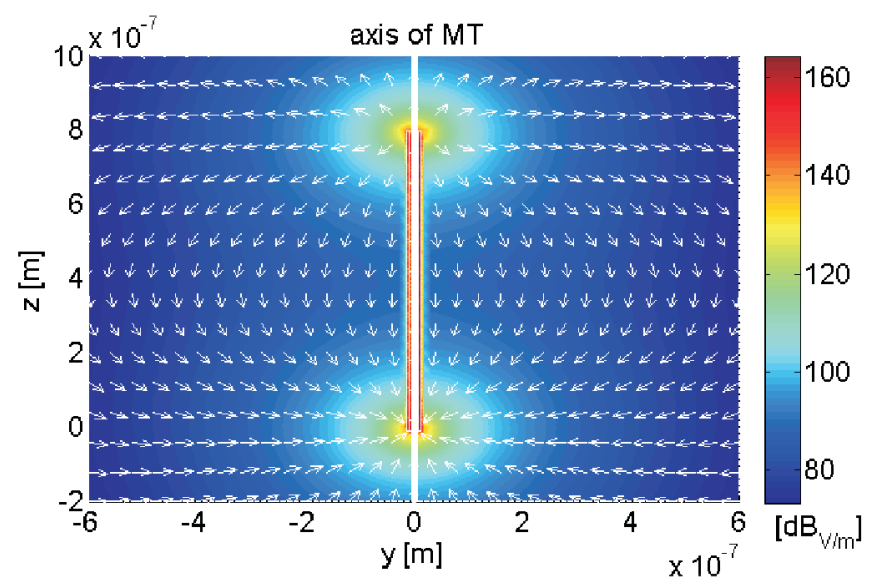

Fig. 6: The excitation in phase

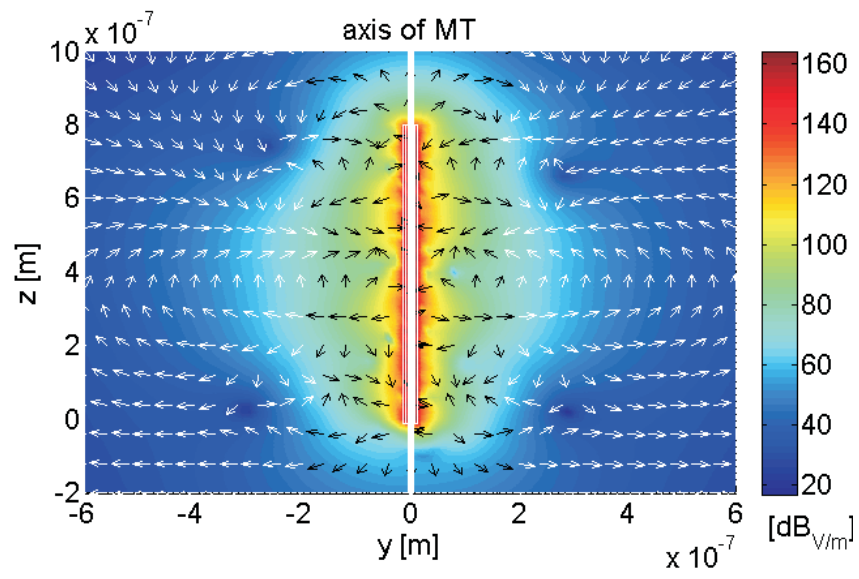

Fig. 7: The random excitation with function "+-" 


\section{Results}

Figs. 6., 7. and 8., and Figs 9. and 10. present a schematic depiction of the EF around MT in the immediate surroundings $\left(6 \cdot 10^{-7} \mathrm{~m}\right)$ and in a distance comparable to the cell size $\left(6 \cdot 10^{-6} \mathrm{~m}\right)$, respectively. Every PT is composed of 100 heterodimers (HDs) and there is a longitudinal shift of each PT of about $4.92 \mathrm{~nm}$ (see Fig. 5a). MT is of $800 \mathrm{~nm}$ in length and is situated in a lossy medium. HDs are excited at a frequency of $1 \mathrm{GHz}$ and the type of excitation is always specified in every

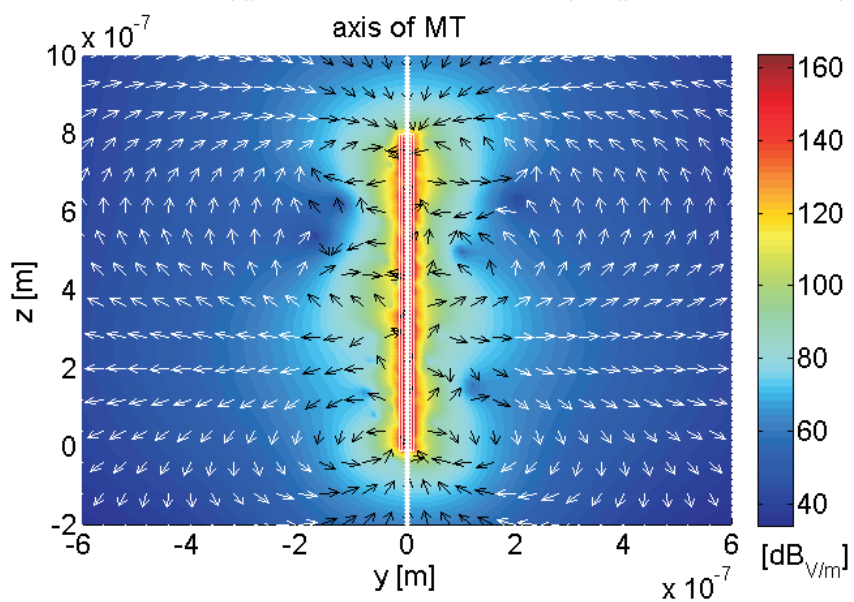

Fig. 8: The random excitation with function " $p$ * $\sin$ "

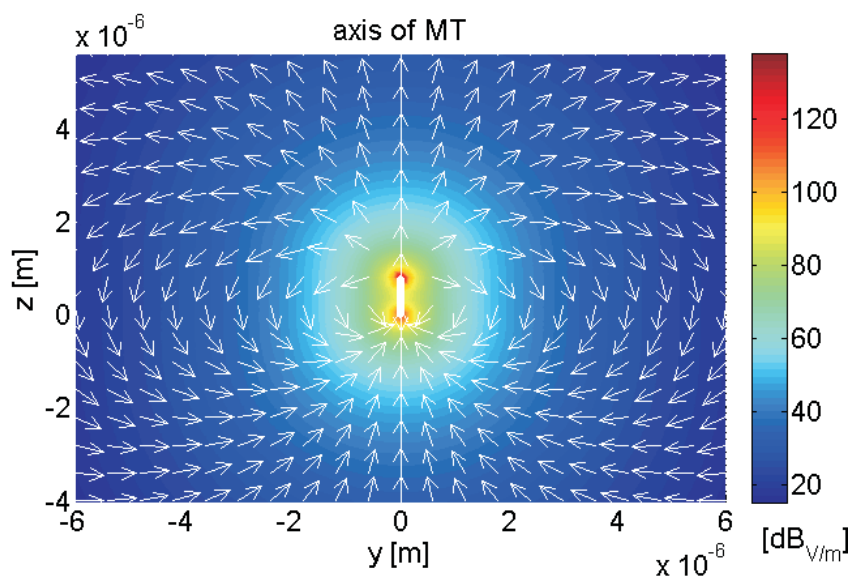

Fig. 9: The excitation in phase

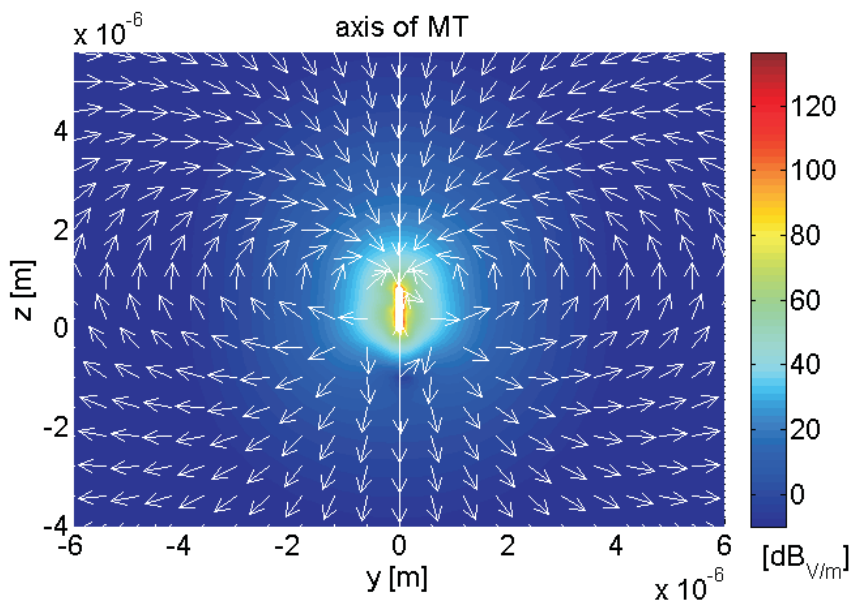

Fig. 10: The random excitation with function "+-"

picture. Arrows in the pictures depict the direction, and the color describes the magnitude of the EF.

\section{Discussion of results}

First, the simplifications we applied in our calculations will be discussed. We used only the modulus of the intensity for the calculating of the field. As mentioned in section 2., this simplification is valid only when the distance between the sources of EMF is very small compared to the EMF wavelength, so at the phase shift and possible interference can be neglected. The excitation frequency was chosen arbitrarily to be $1 \mathrm{GHz}$. Physically it is substantiated in the estimated frequency range of MT oscillations, which was calculated to be in the region from $\mathrm{kHz}$ to $\mathrm{GHz}[7,9,10]$.

We clearly see the difference between the random and in-phase (coherent) oscillations. Although the EF intensity (denoted as IEF in the figures) is higher in the middle of the MT length $(z=0.4 \mu \mathrm{m})$ for random excitation, it falls off faster with distance than in the case of in-phase excitation (Fig. 11). The EF intensity is higher on the poles of MT $(z=0 \mu \mathrm{m}$, $z=0.8 \mu \mathrm{m}$ ) for in-phase excitation than for random excitation (Fig. 12). There is a difference of two orders of magnitude between the EF intensity of randomly excited and in-phase excited EMF in distances larger than ca. $100 \mathrm{~nm}$. Furthermore, random excitation may exhibit local minima of EF in-

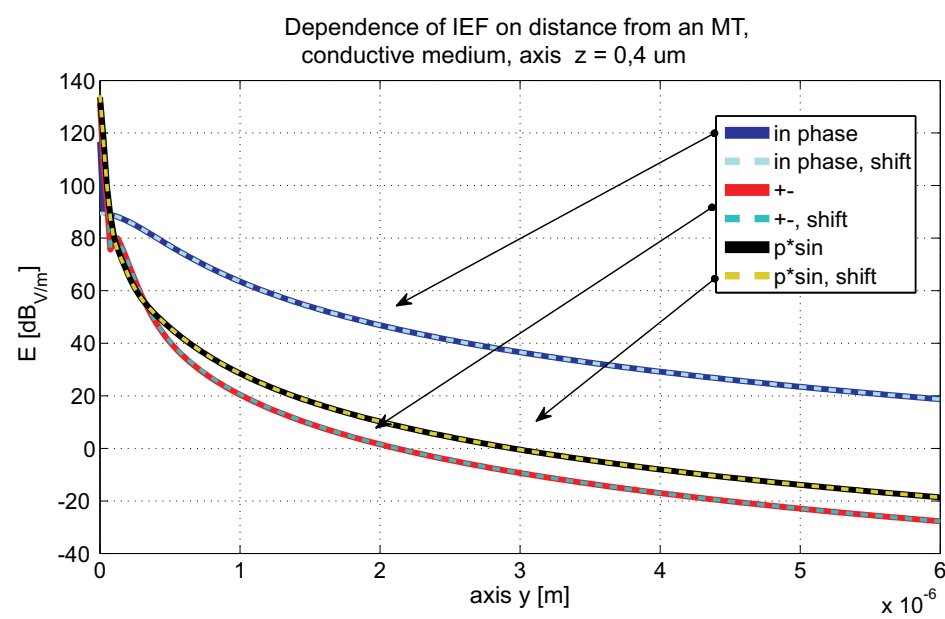

Fig. 11: Dependence of IEF on distance from an MT 


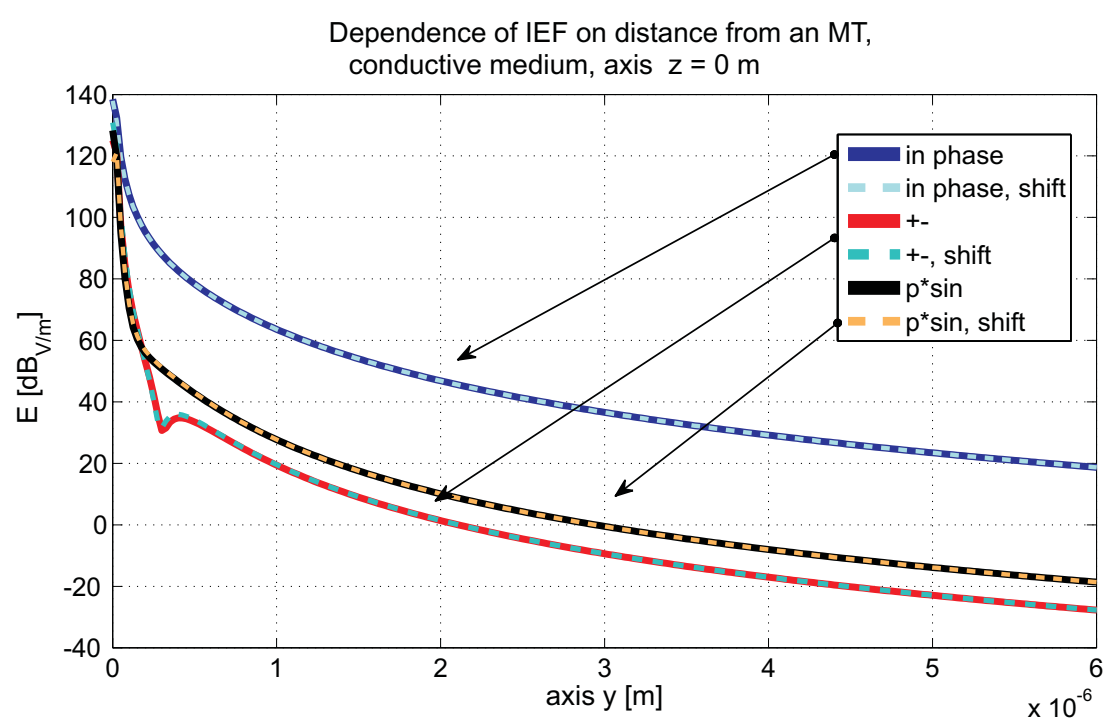

Fig. 12: Dependence of IEF on distance from an MT

tensity, see e.g. Fig. 7 and Fig. 12. In the case of in-phase oscillations, a microtubule acts as one giant dipole. A more realistic calculation should take into account that thermally excited oscillations do not hold the phase not only between oscillators but also in the wave train of a single oscillator. Regarding the in-phase oscillation of a large number of dipoles, the question arises whether there is any physical foundation for phase synchronized oscillations of many dipoles which manifests as a single giant dipole. In fact, similar behavior (giant dipole like oscillation [17]) might be obtained if the oscillation of MT were of zero wave number mode. In the case of weak nonlinear coupling of a chain of oscillating units, energy indeed tends to condense in the lowest mode - reminiscent of the Fermi-Pasta-Ulam problem [18]. The mechanical coupling of individual tubulin heterodimers in MT may be rather strong. Condensation to the lowest mode, if it occurs at all, is therefore more probable due to coupling of individual MTs. A more realistic model of the EMF around MT should also take into account higher longitudinal, circumferential and radial modes and their mutual interactions, as was analyzed in the orthotropic MT model of Wang et al. [10]. The possibility of MT oscillations in physiological conditions was questioned due to viscous damping of oscillations in cytosol [19]. Significant viscous damping does not necessary need to be the case if the slip layer condition is applied for analysis of MT vibrations [20]. This seems to have a solid physical foundation, since the physical properties of cellular water differ significantly from those of bulk water; this also applies for viscous damping. Coherent quantum electrodynamics in matter [20, 21] predicts that a rather large fraction of bulk water and most of cell water is composed of distinct coherent domains which have physical properties (viscosity, density, etc.) different from those of the rest of the water volume. Especially remarkable is that microvolumes of water surrounding biomolecules may exhibit drastically lower damping and viscosity than bulk water [21]. To confirm the existence of MT generated EMF, in vivo measurements are necessary. Either direct nanoelectrical detection or indirect optical detection with the electric field intensity dependent fluorescent dyes would be suitable.

\section{Conclusion}

Microtubules may be a source of endogenous cellular EMF. We have presented here a simple model of MT EMF geometry and properties. Endogenous cellular EMF may contribute significantly to the dynamic and spatial organization of cellular processes and structures [23]. Future work will include in the calculation more realistic field excitation conditions and field distribution according to the modes of the vibrating orthotropic cylindrical shell [10] and the phase.

\section{Abbreviations}

EED - elementary electric dipole, EF - electric field, EMF - electromagnetic field, HD - heterodimer, IEF - intensity of electric field, MT - microtubules, PT - protofilament, PFE point of field evaluation.

\section{Acknowledgments}

The second author acknowledges support from GA CR grant 102-08-H081 and to prof. Vrba and Dr. Pokorný for their guidance and support.

\section{References}

[1] Pelling, A. E., Sehati, S., Gralla, E. B. et al.: Time Dependence of the Frequency and Amplitude of the Local Nanomechanical Motion of Yeast. Nanomedicine: Nanotechnology, Biology, and Medicine, Vol. 1 (2005), p. 178-183.

[2] Pohl, H. A., Braden, T., Robinson, S. et al.: Life Cycle Alterations of the Micro-Dielectrophoretic Effects of Cells, Journal of Biological Physics, Vol. 9 (1981), p. 133-154.

[3] Hölzel, R.: Electric Activity of Non-Excitable Biological Cells at Radio Frequencies, Electro- and Magnetobiology, Vol. 20 (2001), p. 1-13.

[4] Pokorný, J., Hašek, J., Jelínek, F. et al.: Electromagnetic Activity of Yeast Cells in the M Phase. Electro- and Magnetobiology, Vol. 20 (2001), p. 371-396. 
[5] Beloussov, L. V., Voeikov, V. L., Martynyuuk, V. S. eds.: Biophotonics and Coherent Systems in Biology. Springer, 2006.

[6] Fröhlich, H.: The Biological Effects of Microwaves and Related Questions. Advances in Electronics and Electron Physics, Vol. 53 (1980), p. 85-152.

[7] Pokorný, J., Jelínek, F., Trkal, V. et al.: Vibrations in Microtubules, Journal of Biological Physics, Vol. 23 (1997), p. 171-179.

[8] Alberts, B., Bray. J., Lewis, J. et al.: Molecular Biology of the Cell. $3^{\text {rd }}$ edn., Garland, 1994.

[9] Sirenko, Y. M., Stroscio, M. A., Kim, K. W.: Elastic Vibrations of Microtubules in a Fluid, Physical Review E, Vol. 53 (1996), p. 1003-1010.

[10] Wang. C. Y., Ru, C. Q., Mioduchowski, A.: Vibration of Microtubules as Orthotropic Elastic Shells, Physica E, Vol. 35 (2006), p. 48-56.

[11] Novotný, K.: Elektromagnetické pole a vlny: Teorie elektromagnetického pole II. (EMF and waves: Theory of EMF II.) $2^{\text {nd }}$ ed, CTU in Prague, 2001, in Czech.

[12] Havelka, D.: Elektromagnetické pole mikrotubulu (The Electromagnetic Field of a Microtubule) Bachelor thesis, CTU in Prague, 2008, in Czech

[13] Zita, J.: Endogenni elektromagnetické pole v biologických systémech (Endogenous EMF in Biological Systems), Master thesis, FEE CTU in Prague, 2000, in Czech.

[14] Tuszynski, A., Brown, J. A., Carpenter, E. J. et al.: Electrostatic Properties of Tubulin and Microtubules, Proceedings ESA-IEJ Joint Meeting, Chicago, 2002, p. 41-50.

[15] Gabriel, C., Gabriely, S., Corthoute, E.: The Dielectric Properties of Biological Tissues: I. Literature Survey. Phys. Med. Biol., Vol. 41 (1996), p. 2231-2249.

[16] http://www.mathworks.co.uk/matlabcentral/fileexchange $/ 7470$

[17] Fröhlich, H.: Selective Long-Range Dispersion Forces between Large Systems, Physics Letters, Vol. 39A (1972), p. 153-154.

[18] Pohl, H. A.: Do Cells in Reproductive State Exhibit a Fermi-Pasta-Ulam- Fröhlich Resonance and Emit Elec- tromagnetic Radiation, Journal of Biological Physics, Vol. 8 (1980), p. 45-75.

[19] Foster, K. R., Baish, J. W.: Viscous Damping of Vibrations in Microtubules, Journal of Biological Physics, Vol. 26 (2000), p. 255-260.

[20] Pokorný, J.: Viscous Effects on Polar Vibrations in Microtubules, Electromagnetic Biology and Medicine, Vol. 22 (2003), p. 15-29.

[21] Preparata, G.: QED Coherence in Matter, Singapore: World Scientific, 1995.

[22] Zhadin, M. Giuliani, L.: Some Problems in Modern Bioelectromagnetics, Electromagnetic Biology and Medicine, Vol. 25 (2006), p. 227-243.

[23] Pokorný, J., Hašek, J., Jelínek, F.: Endogenous Electric Field and Organization of Living Matter. Electromagnetic Biology and Medicine, Vol. 24 (2005), p. 185-197.

Daniel Havelka

e-mail: haveld1@fel.cvut.cz

Department of Electromagnetic Field

Czech Technical University in Prague

Faculty of Electrical Engineering

Technická 2

16627 Praha, Czech Republic

Michal Cifra

e-mail: cifram1@fel.cvut.cz

Department of Electromagnetic Field Czech Technical University in Prague

Faculty of Electrical Engineering

Technická 2

16627 Praha, Czech Republic

and

Inst. of Photonics and Electronics

Academy of Sciences of the CR

Chaberská 57

18251 Prague, Czech Republic 